\title{
Fusobacterium Nucleatum : An Atypical Cause of Pyogenic Liver Abscess in an Apparently Immunocompetent Patient
}

\author{
Yusoff $A R^{a, b}$, Abd Razak NA ${ }^{a}$, Samsuddin $S^{b}$, Mokhtar $S^{b}$ \\ ${ }^{a}$ Department of Surgery, Faculty of Medicine, Universiti Teknologi MARA \\ ${ }^{b}$ Department of Hepatobiliary Surgery, Selayang Hospital, Batu Caves, Selangor, Malaysia
}

\begin{abstract}
Pyogenic liver abscess is an uncommon but potentially lethal infection if left untreated. Enteric bacteria and anaerobes are the most common causative organisms of pyogenic liver abscess. Although most infections are of a polymicrobial nature, monomicrobial infections are possible. An immunocompromised status and the presence of periodontal disease are the risk factors for liver abscess caused by these rare, monomicrobial anaerobic infections. Here, we report on a successful non-operative management of a case of multiloculated pyogenic liver abscess caused by Fusobacterium nucleatum in a young, healthy, immunocompetent male patient. The diagnosis was established by radiological imaging and was based on a positive aspirate culture, whereas the mainstay of treatment comprised percutaneous drainage and a prolonged course of antibiotics. Thus, the patient was successfully treated with a 2-week course of intravenous imipenem/cilastatin while he was on percutaneous drainage, and he is now doing well.
\end{abstract}

KEYWORDS: Fusobacterium nucleatum, pyogenic liver abscess, immunocompetent patient

\section{INTRODUCTION}

Pyogenic liver abscess (PLA) is an uncommon disease and is associated with unique diagnostic and therapeutic challenges. Its mortality rate ranges from $2 \%$ to $12 \%$, despite advances in its diagnosis and treatment. ${ }^{1}$ Furthermore, its clinical features are variable and depend on the abscess size, general patient health and comorbidities. In majority of the cases, the cause of PLA cannot be determined, ${ }^{2}$ although enteric and anaerobic bacteria are commonly implicated. Most infections associated with PLA are polymicrobial; however, monomicrobial infections do occur as well. ${ }^{3}$ An immunocompromised status and periodontal diseases are among the risk factors that need to be elucidated in the rarer monomicrobial cases of PLA.

Corresponding Author:

Assoc. Prof. Dr. Ahmad Ramzi Yusoff Department of Surgery, Faculty of Medicine, Universiti Teknologi MARA, Jalan Hospital, 47000, Sg Buloh, Selangor, Malaysia

Tel No : +603-6126 7319 .

E-mail : dr_aramzy@yahoo.com
Fusobacterium nucleatum is a rare cause of PLA and usually occurs in immunosuppressed patients. ${ }^{4}$ Here, we report the case of a 27-year-old immunocompetent male patient with multiloculated PLA caused by F. nucleatum and discuss the diagnostic and treatment challenges of achieving favourable outcomes in patients with this disease.

\section{CASE REPORT}

A 27-year-old male patient with unknown medical illnesses or allergies presented with worsening epigastric and right hypochondrial pain for 3 weeks. Further complaints included intermittent fever, chills, anorexia, weight loss and lethargy. However, he did not experience nausea, vomiting, jaundice, tea-coloured urine, pale stool or pruritus. There was no history of abdominal trauma, recent travelling, jungle trekking, river swimming, consuming contaminated food or herbs or dental treatment. Similarly, no history of familial malignancy or autoimmune disease was present.

IMJM Volume 20 No.1, Jan 2021 
He was a college student and lived with his family. Although he denied consuming alcohol, tobacco or illicit drugs, he admitted to having been involved in unprotected homosexual activities over a 10-year period.

Physical examination revealed that he was alert but lethargic, with a body mass index of $21 \mathrm{~kg} / \mathrm{m}^{2}$. He was afebrile and normotensive, with a regular pulse and normal respiratory rate on admission to the ward, but developed a high spiking temperature of $38.8^{\circ} \mathrm{C}$ in the following day. There were no signs of dental caries, scleral icterus, conjunctival pallor or other stigmata associated with chronic liver disease. His abdomen was tender on palpation over the epigastrium and right hypochondrium. Murphy's sign was negative, and no abdominal organomegaly could be detected. Examination of other systems was unremarkable.

Laboratory tests revealed leukocytosis, with a white blood cell count of $39.51 \times 10^{9} / \mathrm{L}$; haemoglobin levels of $11.6 \mathrm{~g} / \mathrm{dL}$; hyponatraemia, with $\mathrm{Na}$ levels of 132 $\mathrm{mmol} / \mathrm{L}$; serum random glucose levels of $8.7 \mathrm{mmol} / \mathrm{L}$ and C-reactive protein (CRP) levels of $10.65 \mathrm{mg} / \mathrm{dL}$. Coagulation profiling revealed a prolonged prothrombin time of 20 s and an activated partial thromboplastin time of $44.7 \mathrm{~s}$ with an international normalised ratio of 1.7 . Liver function tests showed deranged results, with serum albumin $20 \mathrm{~g} / \mathrm{dL}$, total bilirubin $29 \mu \mathrm{mol} / \mathrm{L}$, alkaline phosphatase (ALP) 206 IU/L, alanine aminotransaminase (ALT) $103 \mathrm{IU} / \mathrm{L}$ and aspartate transaminase $95 \mathrm{IU} / \mathrm{L}$. Serologic tests were negative for Burkholderia pseudomallei, amoebiasis, human immunodeficiency virus, hepatitis B virus and hepatitis $C$ virus. Aerobic and anaerobic blood cultures were taken prior to the commencement of antibiotics, and both revealed no growth after a four days incubation period.

An initial transabdominal ultrasound (USG) (Fig. 1 and 2) followed by a triphasic computed tomography (CT) scan (Fig. 3) revealed multiple complex hepatic lesions in both lobes. Multicentric hepatocellular carcinoma was excluded from the differential diagnosis after scrutinising the enhancement pattern of the lesions and confirming the absence of liver cirrhosis.

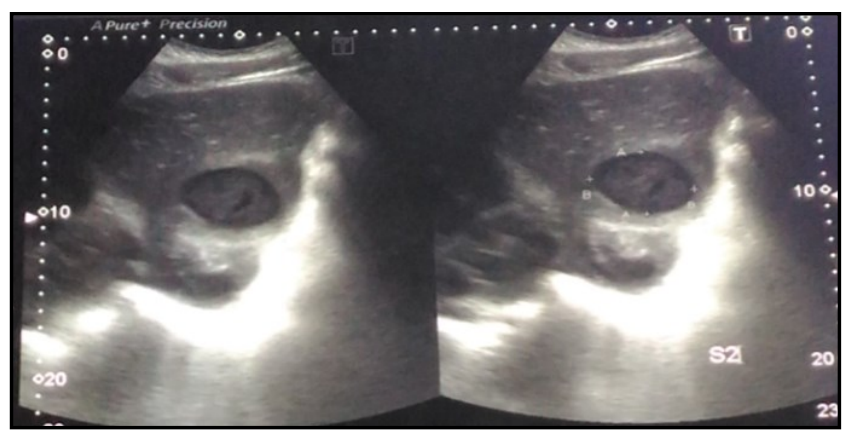

Figure 1: Ultrasound of the liver revealed a well-defined hypoechoic lesion measuring $3.8 \times 5.2 \mathrm{~cm}$ in the left lobe (segment II).

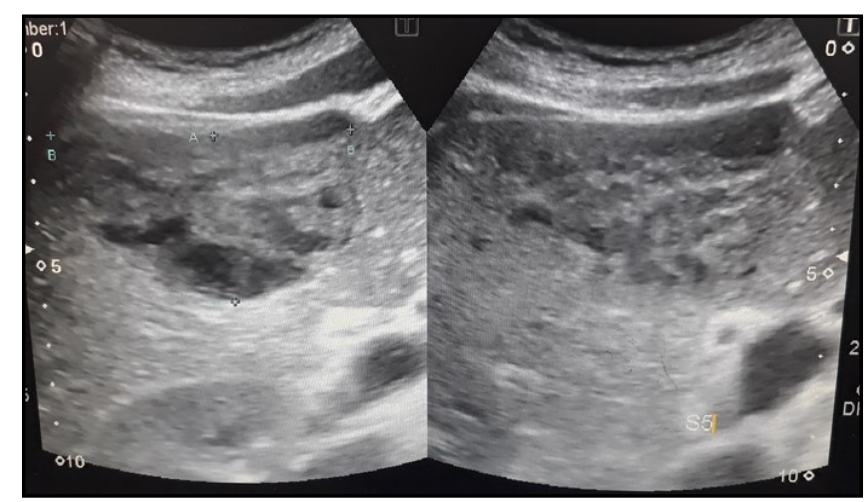

Figure 2: Ultrasound of the liver showing a hypoechoic lesion measuring $3.9 \times 6.9 \mathrm{~cm}$ in the right lobe (segment V)

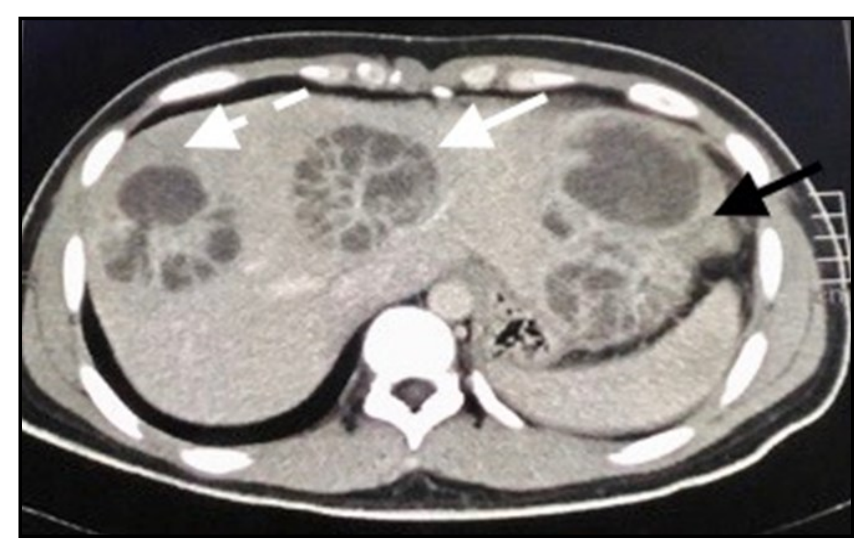

Figure 3: An axial view of abdominal CT showed multiseptated and multiloculated hypodense lesions involving the hepatic lobes bilaterally (black arrow indicates a lesion measuring $12 \times 7.5 \mathrm{~cm}$ in segment II/III of the left lobe; long and dotted white arrows indicate lesions measuring $6.0 \times 7.5 \mathrm{~cm}$ and $6 \times 7.0 \mathrm{~cm}$ in segment $\mathrm{V}$ and VIII of the right lobe respectively).

Purulent material obtained from the largest abscess cavity (segment II of the liver) via an ultrasound-guided percutaneous drainage catheter, tested positive for $F$. nucleatum by the culture and sensitivity test (Tab. 1). 
Culture Fusobacterium nucleatum (anaerobic) isolated.

Table 1: Final culture and sensitivity result of the pus aspirate from the liver abscess.

\begin{tabular}{lccl}
\hline Antibiotics & $\begin{array}{c}\text { Test } \\
\text { method }\end{array}$ & $\begin{array}{c}\text { MIC result } \\
(\mathbf{u g} / \mathbf{m l})\end{array}$ & Result \\
\hline Ampicillin & E-test & 0.016 & Sensitive \\
Clindamycin & E-test & 0.016 & Sensitive \\
Metronidazole & E-test & 0.032 & Sensitive \\
Imipenem & E-test & 0.008 & Sensitive \\
\hline
\end{tabular}

$\overline{\mathrm{MIC}}$ - minimum inhibitory concentration

Empirical intravenous (IV) treatment with cefuroxime $1.5 \mathrm{~g}$ tds and metronidazole $500 \mathrm{mg}$ tds was commenced at admission. Owing to worsening sepsis and suspicion of a possible polymicrobial infection, as well as the culture \& susceptibility testing (Tab. 1), the antimicrobial therapy was upgraded to IV imipenem/ cilastatin $500 \mathrm{mg}$ qid for 14 days. The other abscesses in the right liver were not be drained simultaneously with the left as they were non- and partially liquefied. Successful percutaneous drainage catheters were inserted into the remaining large abscesses three days later. All the catheters were flushed daily with normal saline to maintain patency and to dislodge any fibrinous clot that might hinder effective drainage. The size of each abscess was evaluated on regular intervals with ultrasound to ensure resolution and adequate drainage. The patient was allowed home following removal of all the three drains and completion of IV antibiotic to complete courses of oral cefuroxime axetil $500 \mathrm{mg}$ bid and metronidazole $400 \mathrm{mg}$ tds for 4 weeks.

He showed an uneventful recovery and visited the outpatient clinic at 4 weeks and at 3 months later with subsequent USG of the liver that demonstrated complete resolution of the abscesses (Fig. 4) on the latter occasion.

\section{DISCUSSION}

Liver abscess is a serious health problem associated with a significant mortality rate. 5,6 There are three major forms of liver abscesses, namely, pyogenic, amoebic and fungal. Although amoebic liver abscess is so far the most common form worldwide, the incidence of PLA is higher than that of amoebic liver abscess in developed countries. ${ }^{3,5}$ It usually affects young or middle-aged patients, with a peak in the fourth decade, and shows no gender predilection. ${ }^{2,7}$

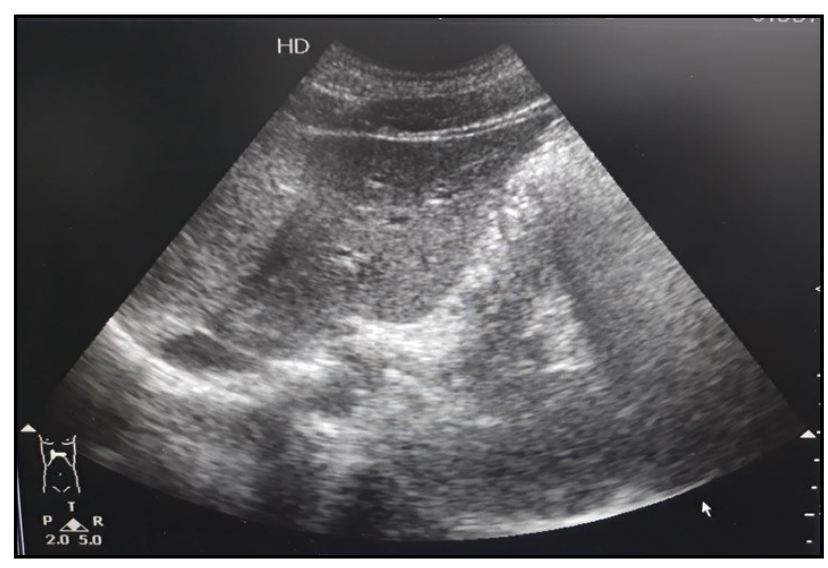

Figure 4: Ultrasound of the liver showed complete resolution of the liver abscess at the 3-month follow-up.

Risk factors for PLA include diabetes mellitus, biliary tract procedures and infection, intra-abdominal malignancy, liver transplantation and immunosuppression, 6,8 but none of these seemed to be apparent in our patient. It is believed that immunodeficient individuals are prone to develop opportunistic or superimposed infections leading to liver abscess. A study by Ohnishi et al. in Japan has reported the association of homosexual activity with the occurrence of amoebic liver abscess; ${ }^{9}$ however, there is no report or clinical study that has confirmed any link between homosexuality and PLA yet.

Polymicrobial involvement is common in PLA infections, with Streptococcus species (29.5\%) and Escherichia coli $(18.1 \%)$ as the two most frequently isolated pathogens. ${ }^{3}$ PLA caused by F. nucleatum has not been widely reported. To date, only 14 cases of liver abscess caused by F. nucleatum have been reported, 12 of which occurred in immunosuppressed individuals. The other two cases were of immunocompetent patients, one male with dental caries and the other in a healthy young male similar to our patient. ${ }^{3}$

Fusobacterium nucleatum, a gram-negative non-spore forming anaerobe, is a commonly observed member of the oropharyngeal microbiota and is mainly found in dental plaques. It may also present in the colorectal or vaginal flora but is rarely detected elsewhere in the body under normal conditions. ${ }^{1}$ The organism is slow growing and is difficult to isolate owing to its sensitivity to 
oxygen. Its tissue-invading capability and virulence are greatly attributed to its endotoxin and FadA adhesin that promote adhesion and co-aggregation. ${ }^{1,3}$

PLA is diagnosed clinically and confirmed via laboratory and imaging studies. Most patients present with fever, chills, right hypochondrial pain, vomiting, dyspnoea and weight loss.2,3 Our patient was afebrile during hospital admission as he was taking antipyretic on his own, but soon developed a high temperature, typical that of multiple abscesses. Biochemical tests often reveal elevated CRP levels, hypoalbuminemia, raised ALP levels and leucocytosis. Measurement of blood glucose levels is mandatory because diabetes mellitus is commonly associated with PLA. ${ }^{4}$ Since the random blood sugar was marginally elevated, and there was no suspicion of insulin-dependent diabetes mellitus from history, our patient was not further investigated for diabetes during his hospitalization. Serological tests for melioidosis, amoebiasis and retroviral disease are routinely performed because these diseases predispose individuals to liver abscess formation. ${ }^{9}, 10$

Abdominal ultrasound is the initial imaging modality, followed by CECT of the abdomen to delineate the extent of the abscess and to exclude unsuspecting liver tumours. ${ }^{2,6}$ Although the abscess is most often solitary and involves the right lobe, multiple lobar involvement is not uncommon, as demonstrated in the case presented here. ${ }^{2}$

Majority of the patients with PLA are successfully treated by image-guided needle aspiration with or without drain insertion. Drainage is essential for large abscesses that are equal to or larger than $5 \mathrm{~cm}$ in size to facilitate resolution as seen in our patient. Open surgical drainage is rarely performed currently and is reserved for failed non-operative treatment. ${ }^{6}$ Fusobacterium sp. is susceptible to most antibiotics, especially to betalactams. ${ }^{7}$ The patient described here was treated with intravenous imipenem/cilastatin $500 \mathrm{mg}$ qid for 14 days while on percutaneous drainage. This antimicrobial therapy was selected based on both the qualitative and quantitative antibiotic susceptibility tests where it had the lowest minimum inhibitory concentration (MIC), among other antibiotics (Tab. 1). He was later discharged and was prescribed oral courses of cefuroxime axetil and metronidazole, similarly according to the sensitivity result, as a 4-6-week course of antimicrobial therapy is usually indicated for complete abscess resolution as evidenced during his follow up.

\section{CONFLICT OF INTEREST}

The authors declare that they have no conflicts of interest.

\section{ACKNOWLEDGEMENTS}

The authors would like to thank the Director General of Health Malaysia for his permission to publish this article and Dr Mohd Rizal Roslan, Head of the Department of Radiology, Selayang Hospital for his expert opinion. This research did not receive any grant or funding from any agency.

\section{REFERENCES}

1. Han YW. Fusobacterium nucleatum: a commensal-turned pathogen. Curr Opin Microbiol 2015; 23:141-7.

2. Mangukiya DO, Darshan JR, Kanani VK, Gupta ST. A prospective series case study of pyogenic liver abscess: recent trands in etiology and management. Indian J Surg 2012; 74(5):385-90.

3. Ahmed Z, Bansal SK, Dhillon S. Pyogenic liver abscess caused by Fusobacterium in a 21-year-old immunocompetent male. World J Gastroenterol 2015; 21(12):3731-5.

4. Wijarnpreecha K, Yuklyaeva N, Sornprom S, Hyman C. Fusobacterium nucleatum: atypical organism of pyogenic liver abscess might be related to sigmoid diverticulitis. $\mathrm{N}$ Am J Med Sci 2016; 8(4):197-9.

5. Nagpal SJ, Mukhija D, Patel P. Fusobacterium nucleatum: a rare cause of pyogenic liver abscess. Springerplus 2015; 4:283.

6. Abbas MT, Khan FY, Muhsin SA, Al-Dehwe B, Abukamar M, Elzouki AN. Epidemiology, clinical features and outcome of liver abscess: a single reference center experience in Qatar. Oman Med J 2014; 29(4):260-3. 
7. Jayasimhan D, Wu L, Huggan P. Fusobacterial liver abscess: a case report and review of the literature. BMC Infect Dis 2017;17(1):440.

8. Kajiya T, Uemura T, Kajiya M, et al. Pyogenic liver abscess related to dental disease in an immunocompetent host. Intern Med 2008;47 (7):675-8.

9. Ohnishi K, Kato Y, Imamura A, et al. Present characteristics of symptomatic Entamoeba histolytica infection in the big cities of Japan. Epidemiol Infect 2004; 132(1):57-60.

10. Wiwanitkit V. Causative agents of liver abscess in HIV-seropositive patients: a 10 -year case series in Thai hospitalized patients. Trop Doct 2005; 35 (2):115-7. 